
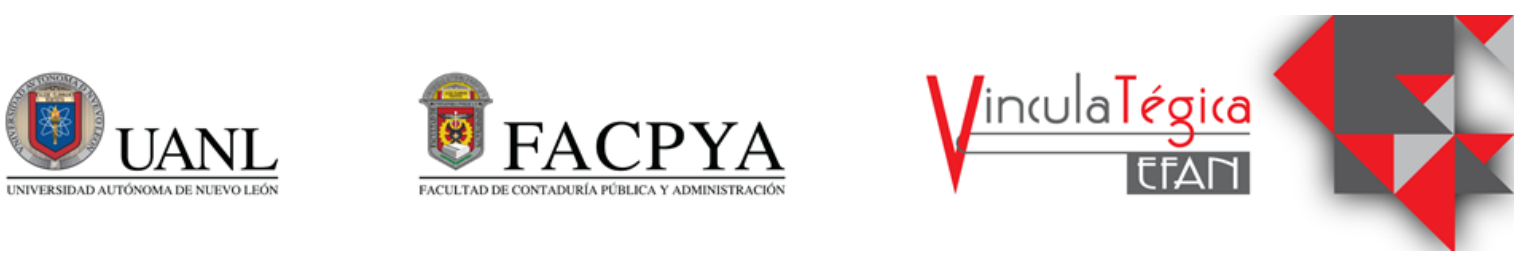

\title{
La influencia que ejerce el capital humano y la prospectiva personal en las empresas familiares del medio rural
}

\author{
Olga Lidia Gutiérrez Gutierrez ${ }^{1}$, Alma Delia Inda $^{2}$ y Gloria Muñoz del Real ${ }^{3}$ \\ ${ }^{1}$ Universidad Autónoma de Baja California, Facultad de Ciencias Administrativas Mexicali, Baja California, \\ México,olgagutierrez@uabc.edu.mx, Calzada de los presidentes y Eje central, Río Nuevo, 21120 Mexicali, \\ B.C., (+52) 6865823334 \\ ${ }^{2}$ Universidad Autónoma de Baja California, Facultad de Ciencias Administrativas Mexicali, Baja California, \\ México, alma.inda@uabc.edu.mx, Calzada de los presidentes y Eje central, Río Nuevo, 21120 Mexicali, B.C., \\ (+52) 6862161589 \\ ${ }^{3}$ Universidad Autónoma de Baja California, Facultad de Ciencias Administrativas Mexicali, Baja California, \\ México,gloria.munoz@uabc.edu.mx, Calzada de los presidentes y Eje central, Río Nuevo, 21120 Mexicali, \\ B.C., (+52) 6861199350
}

Información del artículo revisado por pares

Fecha de aceptación: junio-2021

Fecha de publicación en línea: diciembre-2021

DOI: https://doi.org/10.29105/vtga7.2-6

\section{Resumen}

Las empresas familiares del medio rural en el Valle de Mexicali, Baja California, México, cuentan con algunos desafíos en la identificación de las acciones correctas de análisis para integrar a sus trabajadores. Este trabajo pretende determinar en las empresas dedicadas al comercio, servicio y producción ganadera, la influencia que ejerce el capital humano analizado en relación a las remuneraciones, sean estas por productividad, trabajo en equipo o por habilidades y aptitudes personales, en comparación con la prospectiva personal observada en congruencia con la convivencia empresa-familia, para orientar las acciones de éstas empresas y fortalecer con ello su bienestar económico y social. El estudio fue de corte cuantitativo, analizado por medio del sistema Smart PLS-SEM a través de ecuaciones estructurales, para medir el grado de influencia entre las variables latentes. Uno de los resultados significativos fue que bajo la percepción de los empresarios rurales, la convivencia familiar tiene importancia para el buen desempeño de su trabajo.

\begin{abstract}
Family businesses in rural areas in the Mexicali Valley, Baja California, Mexico, have some challenges in identifying the correct analytical actions to integrate their workers. This work aims to determine in companies dedicated to trade, service and livestock production, the influence exerted by the human capital analyzed in relation to remuneration, be they due to productivity, teamwork or personal skills and abilities, compared to the prospect personnel observed in congruence with the company-family coexistence, to guide the actions of companies included and thereby strengthen their economic and social well-being. The study was quantitative, analyzed by means of the Smart PLS-SEM system through structural equations, to measure the degree of influence between the latent variables. One of the significant results was that under the perception of rural entrepreneurs, family life is important for the good performance of their work.
\end{abstract}

Palabras clave: Empresa familiar, capital humano, 
prospectiva personal

Códigos JEL: M12

\section{INTRODUCCIÓN}

Un desafío que enfrentan las empresas familiares consiste en su crecimiento y continuidad, así como la poca claridad en la división de roles dentro de la organización. En la empresa familiar se conjuga la unión del esfuerzo y el capital familiar, en donde cada uno de los miembros de la familia adquiere roles como el padre, madre, hijos y al mismo tiempo como trabajadores (Gaona, Hernández, Aguilera, Lara 2019). De igual manera para Aronoff y Ward, (2000) la empresa familiar interactúa bajo dos sistemas muy diferentes entre sí: familia y empresa. El sistema familiar genera vínculos, brinda protección, suele ser incondicional, atiende aspectos triviales hacia los integrantes de la familia, por su parte el sistema empresarial se origina de forma contractual, promueve la remuneración que se relacionan con el logro de los objetivos de sus trabajadores, involucrando el aspecto legal, reglamentos, entre otros (Gaona, et. al., 2019).

La falta de entendimiento y comprensión de estos sistemas, así como su grado de diferenciación provoca en las empresas familiares riesgos en su desarrollo y en la colaboración de sus trabajadores. Perret, (2007) establece que si ambos sistemas son identificados claramente, provocará en las empresas familiares un mejor control, crecimiento y estabilidad financiera.

Vadillo (2014) menciona que las empresas familiares cuentan con algunas ventajas por su formación familiar, entre las que se encuentran; valores claros, toma de decisiones, tendencia a pensar a largo plazo y la búsqueda continua de ambientes armónicos, situación que se percibe a través de la prospectiva personal. De igual manera, estas empresas cuentan con ciertas desventajas que ponen en peligro su permanencia, algunas de ellas son; que la relación familiar puede entorpecer la
Key words: Family business, human capital, personal prospective

Códigos JEL: M12.

operación, el trabajo puede generar problemas de familia, no se tratan abiertamente todos los temas y existen pocas posibilidades de discutir. A pesar de contar con grandes ventajas, las empresas familiares presentan según Gon, (2003) relaciones complejas en su interior, donde los valores familiares como la lealtad y protección se mezclan con los valores empresariales, rendimiento y resultados, condición que crea problemas y que ponen en juego la supervivencia de la empresa.

La identificación de acciones que abonen al fortalecimiento y crecimiento de las empresas familiares permitirá su desarrollo, permanencia y efectiva integración de sus trabajadores. Este trabajo tiene como objetivo determinar la influencia que ejerce el capital humano y la prospectiva personal en las actividades de las empresas familiares, para orientar las acciones de éstas y fortalecer con ello su bienestar económico y social. Por ello la pregunta de investigación se cuestiona si, ¿La empresa familiar recibe influencia del capital humano en mayor medida que la prospectiva personal de sus trabajadores?. Para atender esta interrogante se plantean dos hipótesis: $\mathrm{H}^{1}$ : La empresa familiar recibe mayor influencia al remunerar a su capital humano considerando sus habilidades, productividad y trabajo en equipo y $\mathrm{H}_{2}$ : $\mathrm{La}$ empresa familiar recibe mayor influencia al considerar la prospectiva personal de los trabajadores de la organización al mejorar la convivencia familiar y laboral.

\section{MARCO TEÓRICO \\ 2.1 Empresa Familiar}

El desarrollo del emprendimiento se encuentra presente en todo el mundo, tanto en zonas urbanas como rurales, su propósito en el medio rural consiste en reducir la pobreza, aportar a la sustentabilidad ambiental, a la equidad de género, a la revaluación del campo (cultura y su gente), a facilitar la 
descentralización y la participación social, a superar la división rural-urbana y a garantizar la viabilidad de la agricultura campesina. La promoción de las empresas familiares en el campo ha constituido una herramienta para promover la economía local del campo.

La empresarialidad es entendida como "la capacidad que posee todo ser humano para percibir e interrelacionarse con su entorno, influyendo para ello a través de sus competencias empresariales" (BuendíaMartínez y Carrasco, 2013). Las empresas rurales deberán entonces, establecer procedimientos de negocios que orienten su capacidad productiva hacia el desarrollo de sus competencias productivas. El papel que juegan este tipo de empresas es muy importante en el desarrollo de la ruralidad, en este sentido, Molina et al. (2016) menciona que Gallo y Domenec (2004) afirman que la empresa familiar es una empresa cuyo poder de decisión está en una familia, y que las responsabilidades de gobierno y dirección son desempeñadas por algunos de sus miembros sean estos de primera o segunda generación, es decir, que la formalización de la empresa nace en la habilidad de su dirigente el cual debe concretar acciones empresariales que le permitan no solo subsistir sino perdurar como empresa.

El Instituto de la empresa Familiar (2015) menciona que las empresas familiares son menos cortoplacistas y que al existir menor presión en el corto plazo se genera mayor flexibilidad y espacio de maniobra para plantear estrategias y tomar decisiones a largo plazo (visión empresarial). La visión de una familia o empresa es una declaración que indica hacia dónde se dirigen o en qué pretenden convertirse en el mediano y largo plazo, condición que ayuda a enfocar sus recursos, energías y a proyectar una imagen hacia el futuro (Rizo, 2014).

El mantener un equilibrio entre familia y empresa inicia con establecer claramente las líneas de autoridad entre empresa y familia, según Galaz, Yamazaki, Ruiz Urquiza, S.C (2011) la gestión en las empresas familiares cuenta con muchas ventajas, sin embargo existe la necesidad de plantear lineamientos claros que eviten las distorsiones en la participación familiar. Entre los problemas que pueden enfrentar éstas, al no marcar los límites de autoridad se encuentran: nepotismo, confusión de líneas de autoridad, duplicidad de funciones, limitada rendición de cuentas, pobre desempeño, así como la inequidad por parte de los familiares sobre las compensaciones recibidas por su trabajo.

La participación del recurso humano en la empresa familiar, sean estos de la familia o no, representan una ventaja competitiva para este tipo de empresas, es por ello que al incorporar recurso humano se debe tener en cuenta las actitudes y aptitudes de los nuevos trabajadores, debido a que deben otorgar apoyo a quienes cuentan con la responsabilidad de conducir un negocio. López (2010) menciona que hoy en día la formación, la experiencia y las habilidades son factores necesarios para ser seleccionados en un puesto laboral, de igual manera las empresas buscan en sus empleados: actitudes, valores, personalidad y motivaciones, que marque la diferencia entre personal aceptable o no.

Existen factores necesarios, pero no suficientes para seleccionar a un trabajador en una empresa, como por ejemplo; el saber, es decir, identificar los conocimientos, aptitudes y destrezas técnicas del candidato; el saber hacer considerado como las formas metodológicas de proceder en el trabajo; el saber estar, son las pautas y formas de comportamiento individuales y colectivas; y el saber ser son las formas de organización e interacción, estos factores al ser evaluados en los futuros trabajadores de una empresa familiar permiten incorporar empleados que comulguen con la visión de la empresa y que además aporten sus conocimiento, actitudes, aptitudes y valores al logro de los objetivos empresariales (López, 2010).

\subsection{Prospectiva personal}

Las empresas se integran de personas que cuentan con prospectivas personales que les 
permiten confiar en lo que ellas son capaces de hacer, así como también tener especial cuidado en su actuar, una vez identificadas sus limitaciones personales. Es por ello que las organizaciones rurales deben poner especial atención a la convivencia que se genere en sus trabajadores, ya sea en el ámbito familiar o laboral. En este sentido Díaz (2016) declara que existe un alejamiento de los miembros de la familia del hogar por causas de trabajo, condición que resta tiempo a la convivencia, es decir, las empresas familiares deberán cuidar que la convivencia familiar favorezca a las relaciones humanas de sus trabajadores.

Por su parte Gómez y Jiménez
(2015), examinan el concepto de corresponsabilidad familiar desde una perspectiva de armonía familiar y trabajo, los autores identifican a la convivencia familiar como un punto de inflexión para que las labores que el individuo realiza en su trabajo diario sean realizadas de forma armónica. Villarroel y Sánchez, (2002), consideran que en la vida de las personas la familia es considerada como el núcleo fundamental de la sociedad, cuya función es orientar, responder y satisfacer los requerimientos de sus miembros, vinculados con el mundo social. Ambos autores argumentan que la convivencia familiar es un punto importante para realizar mejor una labor.

Los autores Villarruel, Villalobos y Villanueva (2020) consideran que la comunicación en la familia es un aspecto que debe contar con cierto grado de capacitación. En este sentido los autores mencionan que Watzlawick et al. (1995) propone que para poder comprender la importancia de la comunicación se debe tener en cuenta una capacitación sobre el contenido que se transmite y en el nivel relacional que influye sobre la forma en que el contenido es recibido. Este es un factor importante en la dinámica de convivencia familiar, debido a que la comunicación es constante y fluida; condición que favorece el desempeño en la convivencia familiar y laboral. Otro factor relevante es el Poder, debido a la interacción entre los miembros de la familia. El poder es la capacidad de influencia de una persona en otra. Estos factores podrán influir en la mejora de la convivencia familiar, sí estos elementos son fortalecidos a través de capacitaciones donde los miembros de la familia participen.

La convivencia familiar se percibe como presente en todo el quehacer de las empresas familiares, considerada como un reto debido a la riqueza que otorga como comunidad humana (Ginebra, 1997). Una de las estrategias para mejorar la convivencia familiar, consiste en la escenificación de los roles y descripciones de actividades, en orden de funciones que deben cumplir cada uno de los miembros de la empresa familiar. El establecimiento de reglas claras para este propósito debe establecerse desde el inicio y por escrito, siendo estas las claves para lograr el equilibrio en la empresa familiar (Valda, 2010).

Una vez comprendida la importancia de la convivencia familiar y su impacto en las empresas familiares, se distingue también una convivencia laboral, donde Gil (2015) propone que una buena gestión de recursos humanos y la participación activa de los trabajadores es posible en el lugar de trabajo. La Convivencia laboral tiene por objeto contribuir a la optimización de la competitividad de la empresa, otorgando armonía, desarrollo, integración y satisfacción laboral, esto se logra a través de la construcción de mecanismos alternativos que ayuden a la solución de situaciones causadas por conductas de estrés laboral. La integración del personal laboral a través de la convivencia, permite la generación de un ambiente de equidad, autoafirmación y conexión con el individuo, además de mejorar en forma constante su relación con los demás, asimilando y ejecutando códigos sociales que guíen la convivencia dentro o fuera del ámbito laboral (Millas, 2005).

\subsection{Capital Humano}

La fragmentación entre la vida profesional y la vida familiar puede ser la excusa adecuada 
para que se entienda la dinámica entre ambas, el éxito profesional, así como el beneficio económico empresarial no puede estar en contraposición con el beneficio social (López y Grandío, 2005); por lo cual el desenvolvimiento del ser humano tanto en lo profesional como laboral está en constante desarrollo, cada interacción permite mantener un equilibrio en la empresa familiar. Así mismo, también el personal que labora en la empresa debe crecer, desarrollarse y evolucionar en todos los ámbitos: en lo educativo, para incrementar sus conocimientos o desarrollar nuevas habilidades, en lo tecnológico, cultural y social, así como en otros más elementos que le permitan crecer (Naumov, 2018). Todo esto hará que realmente sea un capital humano de la empresa, capaz de enfrentar retos en las distintas áreas que conforman la estructura empresarial.

El capital humano incrementará sus habilidades y/o sus capacidades personales, en función de costos directos e indirectos que la empresa esté dispuesta a invertir, y de los beneficios que les vayan a reportar (Sevilla, 2004). Es por ello que las empresas familiares deciden remunerar las actividades en beneficio del desarrollo intelectual e integral de los miembros de la organización; considerando que estas potencialidades beneficiarán en lo económico. Toda persona que ingresa a una empresa espera crecer en ella en el transcurso de su vida personal y profesional, sobre todo cuando las empresas hoy en día, no solo ofrecen a su personal sueldos y prestaciones competitivas, buen ambiente de trabajo y una estabilidad laboral, sino además el desarrollo profesional y por ende el personal (Naumov, 2018); entre ello se encuentran las remuneraciones que consideran las habilidades personales para lograr así la empatía del capital humano en la organización.

El éxito de la empresa depende en gran medida de la misma, y de las personas que trabajan en ella; los empleados competentes y que tienen una buena disposición pueden contribuir a la productividad y crear una ventaja competitiva, considerando que la productividad es el uso eficaz de la innovación y los recursos para aumentar el agregado añadido de productos y servicios [Organización Internacional del Trabajo (OIT, 2016)]. Si bien, las remuneraciones a los empleados es la recompensa que se les da a los trabajadores que participan de los lineamientos de la empresa, siendo estos motivadores para el compromiso, lealtad y permanencia de los mismos.

Las remuneraciones que la empresa familiar fija a sus empleados y que provocan efectos favorables a las mismas, se puntualizan según la capacidad y la dedicación al trabajo, sin embargo, en la cotidianidad se reconocen algunos criterios para implementarlas, tales como: la dedicación, los resultados, la capacidad, la competencia actual de la remuneración en el mercado laboral, y la necesidad familiar (Dodero, 2005), estas empresas remuneran a su personal con el fin de provocar una productividad que les permita competir con el resto de las organizaciones en su entorno. Establecer una política clara de retribuciones a familiares y personal ayuda a resolver conflictos y diferencias que podrían obstruir la finalidad de la empresa y la armonía familiar (Rizo, 2014).

El capital humano no solo satisface necesidades presentes, sino que también es una inversión susceptible de generar rendimientos pecuniarios y no pecuniarios en el futuro (Sevilla, 2004). Los líderes colaborativos saben obtener el máximo valor de la cultura, la experiencia y habilidades de su equipo y las organizaciones con que interactúan. Para lograrlo deben tener la capacidad de crear relaciones, manejar el conflicto y compartir el control (Archer D, y Cameron A., 2008). Las empresas familiares consideran los trabajos en colaboración que los equipos de trabajo desarrollan a través del cuidado de las habilidades que poseen los trabajadores. 
En toda organización es fundamental un equipo constituido por sus miembros, los beneficios de un buen trabajo en equipo le permite a la organización finalmente cumplir con su misión de manera efectiva, pero hay que tener en cuenta que para que el equipo funcione bien, es necesario tener claro lo que se quiere lograr, y reconocer qué labores puede desarrollar cada uno de los miembros del equipo (Torres G., 2011). Las empresas familiares son por naturaleza una extensión económica de un grupo familia, por lo tanto deberán de preocuparse por implementar los trabajos en equipo con la participación de cada uno de sus miembros; a su vez a los miembros de la organización se les debería permitir hacer sugerencias y tomar iniciativas por su cuenta considerando características estructurales y actitudinales (Barroso A., y Barriuso, C. 2014).

\section{MÉTODO}

Este trabajo busca detectar la influencia que la prospectiva personal y el capital humano ejercen en las empresas familiares del medio rural. Para el análisis estadístico se utilizó el modelo de ecuaciones estructurales (SEM), el cual combina el uso de variables observables y variables latentes. Su estructura considera dos modelos; modelo de medida y modelo estructural, el primero define las relaciones entre las variables observables y las variables latentes; y el segundo explica únicamente las relaciones entre las variables latentes o constructos (Hair, et al., 2013 y Henseler, et $a l ., 2016)$. El enfoque basado en varianzas PLS-SEM se utilizó para este análisis debido a que esta investigación analiza el modelo de forma predictiva (Henseler et al., 2016).

Esta es una investigación explicativa-causal, no experimental, transversal y exploratoria con un enfoque cuantitativo. El enfoque de este estudio evaluó a los empresarios de servicio, comercio y producción ganadera en especies menores ubicados en Cd. Morelos, en el Valle de Mexicali, Baja California, México, considerada esta zona geográfica como una de mayor movimiento económico en la zona rural de la región. El instrumento tipo encuesta que consta de 108 ítems se aplicó de forma directa a 281 MiPyME's rurales. La operacionalización de la variable generó ocho apartados; (1) información general, (2) prospectiva personal, tecnologías de la comunicación (4) empresarialidad, (5) conocimiento del entorno, (6) relación con los clientes, (7) alianzas y (8) recursos humanos.

Los modelos fueron analizados de forma reflectiva, Martínez y Fierro (2007) proponen que para el análisis se realicen pruebas de consistencia interna, validez convergente y estadísticos de colinealidad; de igual manera se examina cada indicador por medio de los resultados que arrojan las cargas factoriales, lo que permite establecer las correlaciones simples entre indicadores y constructos. El análisis factorial exploratorio (AFE) busca maximizar el poder explicativo de un conjunto de variables, Carmines y Zeller, (1979) establecen un valor de carga factorial de 0.707; el análisis realizado permitió discriminar ítems; la variable empresa familiar integrada por 10 ítems, conserva tres (EMP 40, 46 y 47), la Prospectiva personal de 10 ítems, permaneció con cinco (PP 17, 20 y 24) y Capital humano que contaba con cinco ítems, finalizó con 3 (RRH 87, 88 y 89) (Ver tabla 1).

\begin{tabular}{|} 
Tabla 1 Variables de análisis \\
\begin{tabular}{|l|l|c|c|}
\hline $\begin{array}{c}\text { Variables } \\
\text { Latentes }\end{array}$ & \multicolumn{1}{|c|}{ Dimensiones } & Preguntas & Item's \\
\hline \multicolumn{2}{|c|}{ Preguntas de Identificación general } & 14 \\
\hline Empresarialidad & $\begin{array}{l}\text { Empresas } \\
\text { Familiares }\end{array}$ & $\begin{array}{c}\text { EMP } \\
40,46,47\end{array}$ & 3 \\
\hline Prospectiva & Prospectiva & $\begin{array}{c}\text { PP 17 -20 y } \\
24\end{array}$ & 5 \\
\hline Recursos & $\begin{array}{l}\text { Capital } \\
\text { Humano }\end{array}$ & $\begin{array}{c}\text { RRH 87, 88 } \\
\text { y 89 }\end{array}$ & 3 \\
\hline
\end{tabular}
\end{tabular}

Fuente: Elaboración propia del autor

\section{RESULTADOS}

Los modelos de medida son reflectivos debido a que la relación causal va de la variable latente a los indicadores (Bollen, 1989). El modelo estructural considera tres constructos o variables latentes; empresa familiar, prospectiva y capital humano (Ver figura 1). El primero ubica a la empresa en un 
ámbito rural y pretende identificar si la familia involucrada en el negocio cuenta con las mismas metas que la organización y si los empleados familiares son identificados como trabajadores idóneos para el trabajo por el que fueron contratados; el segundo, identificado como prospectiva personal pretende identificar si la convivencia con familiares y trabajadores de la empresa es detectada como una actividad detonadora de relaciones interpersonales que favorezcan al ambiente organizacional; el tercero y último, el constructo de capital humano se enfoca en detectar cuales son las formas de remuneración que la empresa familiar del medio rural considera como factores relevantes que ejercen influencia en la empresa familiar.

Figura 1 Análisis estructural del modelo al tener una mejor convivencia entre los integrantes de la familia en una empresa familiar y el ítem de mayor nivel fue el RRH88 con 0.943 referente a las remuneraciones pagadas en relación a la productividad de los trabajadores.

El índice de fiabilidad compuesta y el Alfa de Cronbach otorga mayor peso a los resultados, debido a que no se asume que todos los indicadores reciben la misma ponderación (Chin, 1998). La fiabilidad compuesta de este modelo determinó para la variable exógena de empresa familiar un valor de 0.856 y para las variables endógenas; prospectiva un valor de 0.884 y capital humano 0.940. El Alfa de Cronbach por su parte mostró valores para empresa familiar de 0.745 , para prospectiva 0.880 y para capital humano de 0.906. Según Nunnally y

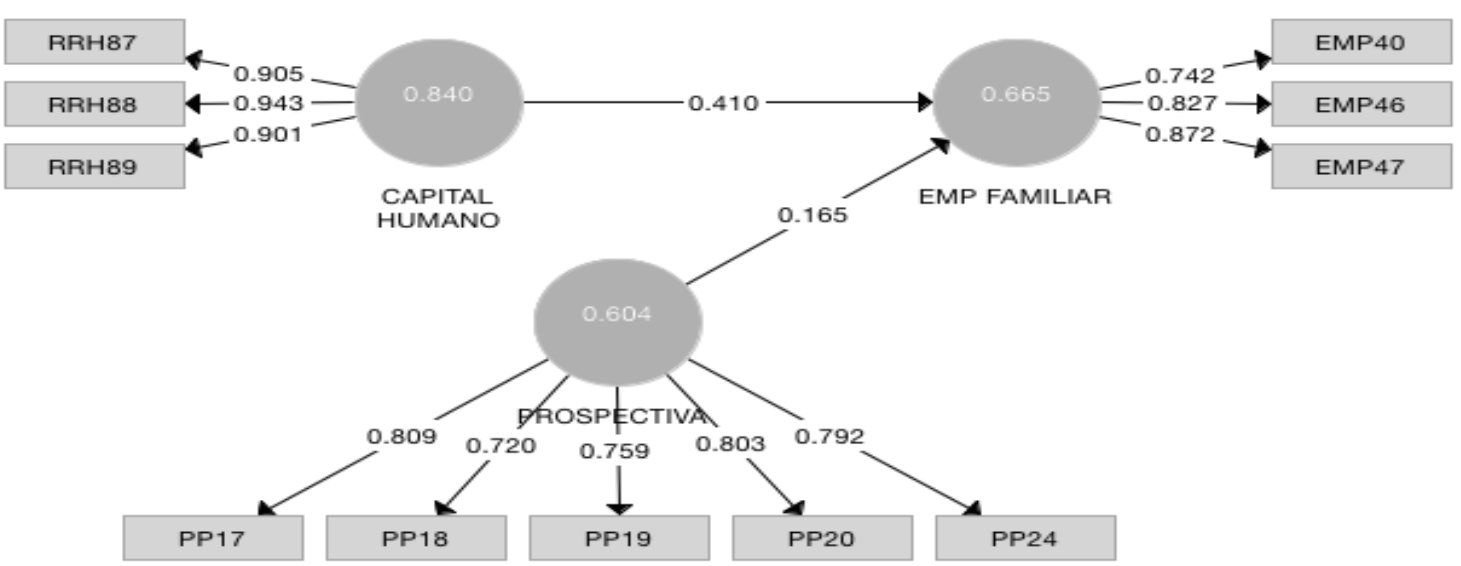

Fuente: Elaboración propia del autor

Bernstein, (1994) los valores obtenidos se consideran como altos para la consistencia interna, debido a que los puntajes se ubican por encima de 0.8 o 0.9 (Ver tabla 2).

en la tabla 2, mostrando los resultados de las pruebas de validez convergente (AVE) y consistencia interna (cargas factoriales, fiabilidad compuesta y alpha de cronbach). Las cargas factoriales de cada uno de los ítems prueban la fiabilidad del constructo, es decir, la correlación simple de los indicadores con respecto al constructo que miden, para Carmines y Zeller (1979) estas cargas deben ser mayores a 0.707. Este modelo presentó como carga factorial menor el indicador PP18 de 0.720 referente a el impacto que se percibe

La validez convergente indica que un conjunto de ítems representa a un único constructo subyacente (Henseler, Ringle y Sarstedt , 2009) su validación se realiza a través de la varianza extraída media (AVE), la cual mide la varianza del constructo explicado a través de sus indicadores (Fornell y Lacker, 1981); el AVE deberá ser > a 0.50. Este modelo determinó un AVE para empresa familiar de 0.665 , para prospectiva $0.604 \mathrm{y}$ 
para capital humano de 0.840 , lo que significa que los constructos están explicados en más del 60\% (Ver anexo 1, tabla 2 Consistencia interna y validez convergente).

Tabla No. 2: Consistencia interna y validez convergente

\begin{tabular}{|c|c|c|c|c|}
\hline & & $\begin{array}{c}\text { Validez } \\
\text { Convergente }\end{array}$ & \multicolumn{2}{|c|}{ Consistencia Interna } \\
\hline & $\begin{array}{c}\text { Cargas } \\
\text { Factoriales } \\
>.70\end{array}$ & $\begin{array}{l}\text { AVE } \\
>.50\end{array}$ & $\begin{array}{l}\text { Fiabilidad } \\
\text { Compuesta } \\
0.70-0.90\end{array}$ & $\begin{array}{l}\text { Alpha de } \\
\text { Cronbach } \\
0.70-0.90\end{array}$ \\
\hline Empresa Familiar & & 0.665 & 0.856 & 0.745 \\
\hline $\begin{array}{l}\text { EMP40. ¿Existe una clara visión del } \\
\text { negocio compartido entre familia y } \\
\text { empresa? }\end{array}$ & $0.742 * * *$ & & & \\
\hline $\begin{array}{l}\text { EMP46. ¿Está claramente identificada la } \\
\text { línea de autoridad para con los familiares? }\end{array}$ & $0.827 * * *$ & & & \\
\hline $\begin{array}{l}\text { EMP47. ¿La incorporación de Familiares } \\
\text { a la empresa se basa en actitudes y } \\
\text { aptitudes? }\end{array}$ & $0.872 * * *$ & & & \\
\hline Prospectiva & & 0.604 & 0.884 & 0.838 \\
\hline $\begin{array}{l}\text { PP17. ¿Le parece importante la } \\
\text { convivencia con su familia? }\end{array}$ & $0.809 * * *$ & & & \\
\hline $\begin{array}{l}\text { PP18. ¿Si mejorará la convivencia con su } \\
\text { familia le ayudaría a mejorar su trabajo? }\end{array}$ & $0.720 * * *$ & & & \\
\hline $\begin{array}{l}\text { PP19. ¿Participaría en capacitaciones para } \\
\text { mejorar su convivencia? }\end{array}$ & $0.759 * * *$ & & & \\
\hline $\begin{array}{l}\text { PP20. ¿La convivencia con el personal de } \\
\text { su empresa es de integración? }\end{array}$ & $0.803 * * *$ & & & \\
\hline $\begin{array}{l}\text { PP24. ¿La convivencia con su familia es } \\
\text { frecuente? }\end{array}$ & $0.792 * * *$ & & & \\
\hline Capital Humano & & 0.840 & 0.940 & 0.906 \\
\hline $\begin{array}{l}\text { RRH87. ¿Al promover y remunerar a sus } \\
\text { empleados usted considera sus } \\
\text { habilidades personales? }\end{array}$ & $0.905 * * *$ & & & \\
\hline $\begin{array}{l}\text { RRH88.¿Al promover y remunerar a sus } \\
\text { empleados usted considera la } \\
\text { productividad? }\end{array}$ & $0.943 * * *$ & & & \\
\hline $\begin{array}{l}\text { RRH89.¿El trabajo en equipo se retribuye } \\
\text { en su empresa? }\end{array}$ & $0.901 * * *$ & & & \\
\hline \multicolumn{5}{|c|}{ P-Value $p<0.05 * * ; p<0.01^{* * * * ;} ;<<1.001$} \\
\hline Fuente: Elaboración Propia del Autor & & \multicolumn{3}{|c|}{$\begin{array}{l}\text { aceptable) lo que significa que no existe } \\
\text { multicolinealidad; sin embargo el ítem RRH } \\
88 \text { muestra un valor de } 5.728 \text { por lo que el } \\
\text { cual permanece en el modelo debido a la } \\
\text { importancia de la interrogante (Ver tabla } 3 \text { ). }\end{array}$} \\
\hline
\end{tabular}

Los resultados muestran los valores de VIF en cada uno de sus indicadores (Empresa familiar, capital humano, prospectiva personal) no superan el valor de cinco (como

Tabla No. 3 Estadísticos de colinealidad (VIF)

\begin{tabular}{|c|c||c|c||l|l|}
\hline ITEM'S & VIF & ITEM'S & VIF & ITEM'S & VIF \\
\hline EMP 40 & 1.295 & PP 17 & 1.976 & RRH 87 & 3.972 \\
\hline
\end{tabular}




\begin{tabular}{|c|c|c|c|c|c|}
\hline EMP 46 & 1.755 & PP 18 & 1.624 & RRH 88. & 5.728 \\
\hline EMP 47 & 1.980 & PP 19 & 1.924 & RRH 80 & 2.325 \\
\hline & & PP 20 & 1.738 & & \\
\hline & & PP 24 & 2.024 & & \\
\hline
\end{tabular}

La tabla 4 muestra un análisis adicional de validez discriminante y convergente, misma que es considerada como una característica complementaria donde el constructo solo mide las características del concepto que pretende representar y no de otro constructo (Sánchez-Pérez, y JiménezCastillo, 2013). Este modelo cumple con la validez discriminante debido a que presenta valores mayores en cada uno de sus indicadores relacionados con el mismo que con los demás.

Tabla No. 4: Validez Discriminante y Convergente

\begin{tabular}{|l|c|c|c|}
\hline & $\begin{array}{c}\text { Empresa } \\
\text { Familiar }\end{array}$ & Prospectiva & $\begin{array}{c}\text { Capital } \\
\text { Humano }\end{array}$ \\
\hline $\begin{array}{l}\text { Empresa } \\
\text { Familiar }\end{array}$ & 0.707 & & \\
\hline Prospectiva & 0.436 & 0.713 & \\
\hline $\begin{array}{l}\text { Capital } \\
\text { Humano }\end{array}$ & 0.577 & 0.519 & 0.872 \\
\hline
\end{tabular}

Fuente: Elaboración Propia del Autor

Para confirmar el rechazo o aceptación de la hipótesis se evaluó el estadístico de coeficiente de regresión estandarizado Path, posteriormente se analizó el nivel de significancia de $t$ de Student y su relación con el valor de $P$-value, de igual manera se presenta el tamaño del efecto del modelo estructural en su conjunto indicado a través de la $\mathrm{F}^{2}$. El coeficiente Path mide el grado de variación producida en la variable dependiente por cada una de las variables independientes (Wright, 1923), este modelo presentó valores para $\mathrm{H}^{1}$ de 0.410 y para $\mathrm{H}^{2}$ de 0.1650. Para Chin, (1998) cada relación entre constructos los valores deberían considerar un valor mínimo de 0.2 , lo que significa que los coeficientes de Path del modelo cumplen parcialmente con el estándar (Ver tabla 5).
El P-Value confirma la aceptación o rechazo de las hipótesis, este modelo a mostrado para $\mathrm{H}^{1}$ de 0.000 y para $\mathrm{H}^{2} 0.084$, lo que significa que el capital humano influye directamente en las empresas familiares y que la prospectiva influye en menor medida a la empresa familiar, por consiguiente los resultados de la aplicación de los algoritmos PLS concluyen que la $\mathrm{H}^{1}$ es aceptada y la $\mathrm{H}^{2}$ es rechazada. Además la prueba de $\mathrm{F}^{2}$, considera un efecto en $\mathrm{H}^{1}$ de 0.179 y en $\mathrm{H}^{2}$ de 0.029 , por lo cual el modelo muestra un efecto moderado (Ver tabla 5).

Tabla 5 Resultado de la Prueba de Hipótesis

\begin{tabular}{|l|c|c|c|c|}
\hline Hipótesis & $\begin{array}{c}\text { Path } \\
\text { Valor } \\
\beta\end{array}$ & $\begin{array}{c}\text { P- } \\
\text { Value }\end{array}$ & $\mathrm{F}^{2}$ & $\begin{array}{l}\text { Aceptada/ } \\
\text { Rechazada }\end{array}$ \\
\hline $\begin{array}{l}\mathrm{H}^{1} \text { : Capital } \\
\text { Humano } \\
\rightarrow \text { Empresa } \\
\text { Familiar }\end{array}$ & 0.410 & 0.000 & 0.179 & Aceptada \\
\hline $\begin{array}{l}\mathrm{H}^{2}: \\
\text { Prospectiva } \\
\rightarrow\end{array}$ & 0.165 & 0.084 & 0.029 & Rechazada \\
$\begin{array}{l}\text { Empresa } \\
\text { Familiar }\end{array}$ & & & & \\
\hline
\end{tabular}

Para determinar la capacidad y relevancia predictiva del modelo así como el criterio de ajuste del Modelo Global se realizaron las pruebas de $\mathrm{R}^{2} \mathrm{Q}_{2}$ y $\mathrm{SRMR}$ aplicadas a la $\mathrm{H}^{\text {; }}$ los valores obtenidos en $\mathrm{R}^{2}$ fue de 0.172 considerada como débil (Chin 1998), es decir el capital humano tiene un efecto débil sobre la empresa familiar y es explicada en un $17 \%$. La $\mathrm{Q}^{2}$ presenta un valor de 0.144 , lo que significa que el capital humano es medianamente predictivo (Geisser, 1974). Actualmente el único criterio de ajuste del modelo global es la normalización de $\mathrm{R}^{2}$ media residual (SRMR), Henseler, Ringle y Sarstedt (2015) menciona que un modelo correctamente especificado debe obtener un valor superior a 0.06 , este modelo estructural a presentado un SRMR de 0.083 , lo que significa que el modelo es predictivo en relación al capital humano y su influencia sobre la empresa familiar (Ver tabla 6). 
Tabla 6 Capacidad predictiva y adaptación del

\begin{tabular}{|c|c|c|c|}
\hline \multicolumn{4}{|c|}{ modelo } \\
\hline Dimensión & $\mathrm{R}^{2}$ & $\mathrm{Q}^{2}$ & SRMR \\
\hline Capital Humano & 0.179 & 0.144 & 0.083 \\
\hline
\end{tabular}

\section{CONCLUSIONES}

Se concluye que las empresas familiares reciben mayor influencia del capital humano en comparación con la prospectiva personal. Lo que significa que las remuneraciones relacionadas con las habilidades, la productividad y el trabajo en equipo que realizan los trabajadores conduce a su mayor desenvolvimiento, condición que beneficia a la organización. La remuneración es considerada como un motivador para el compromiso, lealtad y permanencia del personal, se sugiere según el autor Dodero (2005) la consideración de algunos criterios para su implementación, tales como: dedicación, resultados, capacidad y la competencia actual de la remuneración en el mercado laboral.
De igual manera la remuneración debe considerar la productividad de los trabajadores, según el autor Rizo (2014) esta consideración ayuda a resolver conflictos y diferencias entre el personal de la empresa. Adicional, la empresa debe tomar en cuenta para la asignación de una remuneración efectiva, el reconocimiento del trabajo en equipo, para ello Torres (2011) especifica que primero se deberá identificar la actividad de trabajo y posteriormente el desarrollo que cada miembro del equipo ejecuta. Es así que, las empresas familiares deberán vigilar la integración de los trabajadores al logro de los objetivos empresariales, realizando acciones pertinentes para fortalecer su organización. Sin embargo, la convivencia empresa-familia no debe dejarse de lado, debido a que los trabajadores de este tipo de empresas deben alinearse a la orientación empresarial en el sentido económico y de relaciones sociales.

\section{REFERENCIAS}

Archer D, y Cameron A., (2008). Collaborative Leadership: Building Relationships, Handling Conflict and Sharing Control. 2da Ed. Routledge.

Aronoff y Ward, (2000). Aronoff, C., \& Ward, J. (2000). La sucesión en la empresa familiar un nuevo líder un nuevo héroe (Primera ed.). México: México McGraw-Hill Interamericana.

Barroso A., y Barriuso, C. (2014). Las empresas familiares. https://www.unex.es/conoce-lauex/centros/eia/archivos/iag/2014/2014_04\%20Las\%20empresas\%20familiares.pdf

Bollen, K. (1989). Structural Equation with latent variables. Estados Unidos, North Carolina: John Wilet \& Sons.

Carmines, E. y Zeller, R. (1979). Reliability and validity assessment. N. 07-017, Sage University Paper Series on Quantitative Applications the Social Sciences. Beverly, Estados Unidos: Sage.

Chin, W. (1998). Issues and Opinion on Structural Equation Modeling, MIS Quarterly, 22 (1) March: vii-xv.

Díaz, L (2016). Mejorando la comunicación familiar, disminuyendo los conflictos, Proyecto de intervención para mejorar la comunicación familiar. Tesis de grado en educación social. Universidad de Málaga, Facultad de Ciencias de la Educación Trabajo. https://riuma.uma.es/xmlui/bitstream/handle/10630/12698/D\%C3\%ADaz\%20Barbero_TFG Educaci\%C3\%B3n\%20Social.pdf?sequence $=1$

Dodero, S. (2005). Investigación sobre la empresa familiar en latinoamérica. Instituto de la Empresa Familiar.

http://www.biblioferrersalat.com/media/documentos/Investigacion\%20sobre\%20la\%20Emp resa\%20Familiar\%20en\%20Latinoamerica\%20(2).pdf 
Enciso, E. Cardona, C. Ruiz, F. (2000). Estudio prospectivo del perfil del psicólogo organizacional competitivo para el siglo XXI. Proyecto Docente. Universidad Católica de Colombia. Bogotá.

Feinstein, H. (2002). El trabajo en equipo en las organizaciones. Recuperado en agosto 15, 2002 disponible en: http//:www.-hFeinstein.com.ar/ articul/ed31999.html.

Fornell, C. y Lacker, D. F. (1981) Evaluating structural equation model with unobservable variables and measurement error, Journal of Marketing Research, 18 (1), 39-50

Galaz, Yamazaki, Ruiz Urquiza, S.C (2011). Gestión de las empresas familiares Cómo lograr la ejecución de la visión familiar, Deloitte, Boletín Gobierno Corporativo https://www2.deloitte.com/content/dam/Deloitte/mx/Documents/risk/GobiernoCorporativo/gestion-empresas-familiares.pdf

Gaona L., Hernandez, R., Aguilera, G., Lara P. (2019). Necesidad de un plan para identificar al sucesor de la empresa familiar. Global Conference on Business and Finance Proceedings, Volume 14, Number 2.

Geisser, S. (1974). A predictive approach to the random effects model. Biometrika, 61, 101-107.

Gil, M. I. (2015), Efectos de la interacción humana en la productividad y eficiencia de las empresas, Universidad militar nueva Granada, Especialidad alta gerencia. https://repository.unimilitar.edu.co/bitstream/handle/10654/13588/LA\%20CONVIVENCIA \%20EMPRESARIAL.pdf;jsessionid=A5A805CDBF92A5888D14FAB98C6819E0?sequen $\underline{\mathrm{ce}=1}$

Ginebra, J. (1997). Las empresas familiares, sudirección y su continuidad. 1ra Edición. Panorama editorial.

Gómez, V. y Jiménez, A. (2015), Corresponsabilidad familiar y el equilibrio trabajo-familia: medios para mejorar la equidad de género, Polis [En línea], 40, consultado el 19 abril 2019. URL : http://journals.openedition.org/polis/10784

Gon, P. (2003), Problemas de las empresas de familia desde la perspectiva de los Recursos Humanos, un estudio de campo. Revista de investigación académica, No. 11, pp 101-112, https://dialnet.unirioja.es/servlet/articulo?codigo $=3330725$

Hair, J., Ringle, C. M., Sarstedt, M. (2013), Editorial-Partial Least squares structural equation modeling: rigorous application, better results and higher acceptance. Long range planning, $46(1: 2-12)$.

Henseler, J., Hugona, G., Ash Ray, P. (2016), Using PLS path modeling in new technologic y research: updated guidelines. Industrial Management y Data Systems, 116 (1:20).

Henseler, J., Ringle, C.M, y Sarstedt, M. (2009). The use de partial least squares path modeling in international marketing. Advances in International marketing, 20, 277-320. https://repositorio.cepal.org/bitstream/handle/11362/44148/1/S1800707 es.pdf

Instituto de la empresa Familiar (2015). Una visión de la empresa familiar excelente. https://assets.kpmg/content/dam/kpmg/pdf/2015/11/vision-empresa-familiar-excelente.pdf

Lopez A, y Grandío A. (2005). Capital humano como fuente de ventajas competitivas. Algunas reflexiones y experiencias. Gesbiblo, S.L. Ed. España.

López, J. R. (2010), La selección de personal basada en competencias y su relación con la eficacia organizacional, PERSPECTIVA, núm. 26, pp. 129-152 Universidad Católica Boliviana San Pablo Cochabamba, Bolivia. https://www.redalyc.org/pdf/4259/425941230007.pdf

Marcoulides, G. y Saunders, C. (2006). "PLS: A Silver Bullet?" MIS Quarterly, (30: 2).

Martínez, M. y Fierro, E. (2007) "Aplicación de la técnica PLS-SEM en la gestión del conocimiento: un enfoque técnico práctico", Revista Iberoamericana para la Investigación y Desarrollo Educativo. Consultado 29 enero 2020 pagina web file:///Users/gloriamunozdelreal/Downloads/DialnetAplicacionDeLaTecnicaPLSSEMEnLaGestionDelConocimie-6308426\%20(5).pdf

Millas, V. (2005), Guía Práctica para la elaboración de un programa de integración laboral de personas con discapacidad. Universidad Gabriela Mistral. Acción RSE. 
https://www.incluyeme.com/wp-content/uploads/2019/01/Guia-Practica-Empresas-

Integracion-Laboral-Personas-Discapacidad.pdf

Molina, P. A.; Botero, S.; Montoya, J. N. (2016). Empresas de familia: conceptos y modelos para su análisis Pensamiento \& Gestión, núm. 41, pp. 116-149 Universidad del Norte Barranquilla, Colombia

Naumov S. (2018). Gestión e innovación total del capital humano. Primera Edición E-Book. Patricia Educación.

Nunnally, J. y Bernstein, I. (1994). Psychometric theory (3a Ed.). Nueva York, Estados Unidos: McGraw-Hill.

Perret, (2007). Perret, E. (2007). Innovación en las empresas familiares. Contaduría Pública no. 418, 24-25.Rizo, M. (25 de Agosto de 2015). 7 tips para formar al sucesor. Obtenido de MARIO RIZO: http://www.mariorizo.com/7-tips-para-formar-al-sucesor/

Rizo, M (2014). Visión, misión y valores: esencia de la empresa familiar, https://www.forbes.com.mx/vision-mision-y-valores-esencia-de-la-empresa-

familiar/\#: :text=La\%20visi\%C3\%B3n\%20de\%20una\%20familia,el\%20mediano\%20y\%2

0largo \%20plazo.\&text $=$ Lo $\% 20 \mathrm{~m} \% \mathrm{C} 3 \% \mathrm{~A} 1 \mathrm{~s} \% 20 \mathrm{importante} \% 3 \mathrm{~A} \% 20$ ayuda\%20a,para\%201 ograr\%20una\%20meta\%20com\%C3\%BAn.

Rizo, M. (2014) ¿Cuánto les pago a mis familiares?, nota periodística revista Forbes, febrero 19, 2014 @ 8:00 am,https://www.forbes.com.mx

Roldán, J.L. \& Sánchez-Franco, M.J. (2012). Variance-Based Structural Equation Modeling: Guidelines for Using Partial Least Squares. Research methodologies, innovations and philosophies in software systems engineering and information systems , 193.

Sánchez-Pérez, M. y Jiménez-Castillo, D. (2013), la Validez del Instrumento de medida. En Sarabia, F. J. (coord.) Métodos de Investigación social y de la empresa (387-420). Madrid: Pirámide.

Sánchez, X., \& Villarroel, G. (2002). Relación Familia y Escuela: Un Estudio Comparativo en la Ruralidad. Recuperado el 12 de Junio de 2015, de Estudios Pedagogicos: http://www.scielo.cl/scielo.php?script=sci_arttext\&pid=S0718-07052002000100007

Sevilla C, (2004). El capital humano y su contribución al crecimiento económico. Ediciones de la Universidad de Castilla-La Mancha, Cuenca, 2004.

Torres G., (2011). Administración y gestión de empresas familiares. Trabajo de Investigación. Universidad Nacional de Cuyo, Facultad de Ciencias Económicas Mendoza, Agosto de 2011.

Vadillo, S. (2014). Desventajas y ventajas de las empresas familiares, Universo PYME, http://ri.uaemex.mx/bitstream/handle/20.500.11799/31754/secme-18664.pdf?sequence=1

Valda, J. C. (2010). Cuando inicias un negocio, es común que tus compañeros de aventura sean tus propios parientes. http://jcvalda.wordpress.com/2010/10/02/cuando-inicias-un-negocio-escomun-que-tus-companeros-de-aventura-sean-tus-propios-pariente/

Villarruel, Villalobos y Villanueva (2020) Orientando para facilitar el cambio en las familias: Un modelo de intervención. Revista Electrónica Educare On-line versión ISSN 1409-4258 Print versión ISSN 1409-4258. Educare vol.24 n.2 Heredia May./Aug. 2020 Epub May 01, 2020, http://dx.doi.org/10.15359/ree.24-2.9

Wright, S. (1923). The theory of path coefficients: A reply to Niles's criticism. Genetics, 8, 239-255 